\title{
Primary Hodgkin's disease of the lung: case report
}

\author{
STEVE NELSON, DAVID PRINCE, PETER TERRY \\ From the Respiratory Division, Department of Medicine, Johns Hopkins Hospital, Baltimore, Maryland, \\ USA
}

Pulmonary disease in Hodgkin's disease is common, occurring in $15-40 \%$ of patients. ${ }^{1}$ Most often it is seen in the setting of advanced stage IIIB or IV disease..$^{23}$ Parenchymal disease without hilar or mediastinal node enlargement is thought to be an uncommon presentation, but with stage III and IV disease lung manifestations without adenopathy have been noted with surprising frequency. McDonald reviewed 122 patients with advanced disease including lung lesions and found 31 to have parenchymal disease without adenopathy. ${ }^{2}$ Nevertheless, he noted no case of purely parenchymal disease.

Isolated Hodgkin's disease affecting the lung without hilar adenopathy or disseminated disease is rare $^{45}$ and is referred to as primary pulmonary Hodgkin's disease. As there is a considerable amount of lymphoid tissue in the peribronchial areas, a primary origin in the lung can be explained. We wish to describe the second documented case of primary pulmonary Hodgkin's disease.

\section{Case report}

A 34-year-old white man with a one-year history of weight loss and malaise and recent appearance of blood-tinged sputum was admitted for evaluation. A chest radiograph at that time showed a mass in the left upper lobe (fig 1). The patient had an eight-month history of a non-productive cough, but denied dyspnoea, chest pain, fever, and night sweats. He had had no known exposure to harmful industrial or environmental agents and was a non-smoker.

On examination there was no palpable lymphadenopathy. The left anterior chest was dull to percussion. The remainder of the physical examination showed nothing abnormal.

Laboratory investigation showed a normal haemoglobin concentration and white blood cell count and no abnormality on examination of a blood film. The erythrocyte sedimentation rate was $55 \mathrm{~mm}$ in one hour. Liver function tests, serum electrolyte estimations and urine analysis gave normal results. Intermediate-strength purified protein derivative evoked no response and there was anergy to Candida, mumps virus, and Trichophyton antigens. Computed tomography of the chest showed a large mass in the left upper lobe and patent bronchi. There was no hilar adenopathy and the mediastinum was normal.

The patient underwent fibreoptic bronchoscopy and no obstruction of the bronchi was seen. Multiple brushings for

Address for reprint requests: Dr Peter B Terry, Johns Hopkins Hospital, 60() North Wolfe Street, Brady 418, Baltimore. Maryland 21205 , USA.

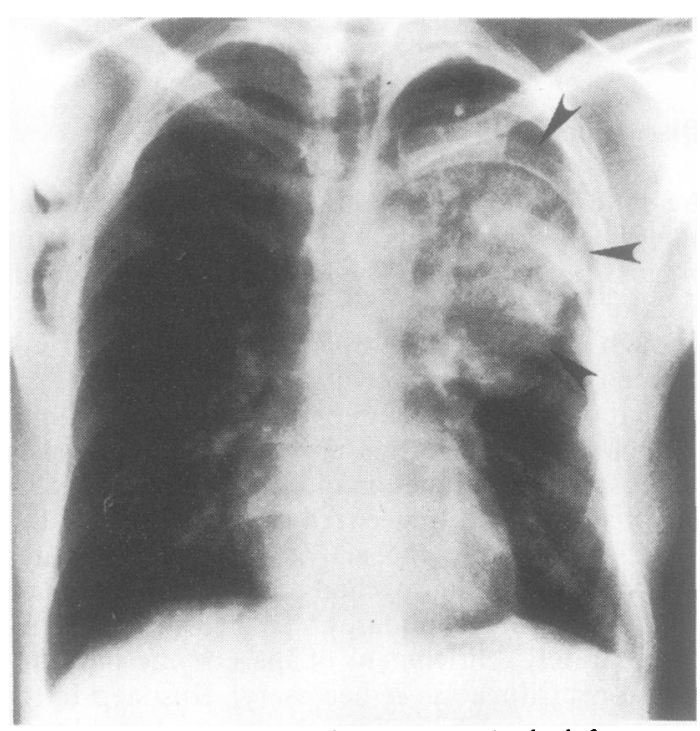

Fig 1 Chest radiograph showing mass in the left upper lobe (arrows).

acid-fast bacilli and fungi and cytological examination gave 3 negative results. A percutaneous lung biopsy was performed but no definitive diagnosis was obtained.

A left thoracotomy was performed, which showed a

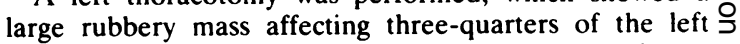
upper lobe. No hilar adenopathy or mediastinal disease $D$ was seen. The remainder of the lung appeared normal. No attempt was made to resect the mass. Needle biopsy $\mathrm{N}$ specimens of the mass were obtained which showed pre- or dominantly histologically normal lymphocytes, plasma $\mathrm{N}$ cells, and eosinophils with microscopic foci of necrosis and fibrosis. Within the infiltrate were atypical mononuclear $\sigma$ and multinuclear cells, including mononuclear variants of Reed-Sternberg cells (fig $2 \mathrm{a}$ ). Reed-Sternberg cells $\stackrel{0}{\mathrm{C}}$ characteristic of Hodgkin's disease were seen (fig 2b). $\mathbb{\oplus}$ After the results of the lung biopsy became available a? bone scan, bone marrow aspiration and biopsy, $\underset{T}{T}$ liver-spleen scan, and lymphangiogram were performed. All gave normal results.

The pathological appearances were felt to be most $\stackrel{\mathbb{Q}}{\Phi}$ consistent with nodular sclerosing Hodgkin's disease. The patient was staged as $I_{\mathrm{E}}$ and received six courses of chemotherapy with mechlorethamine, 8 


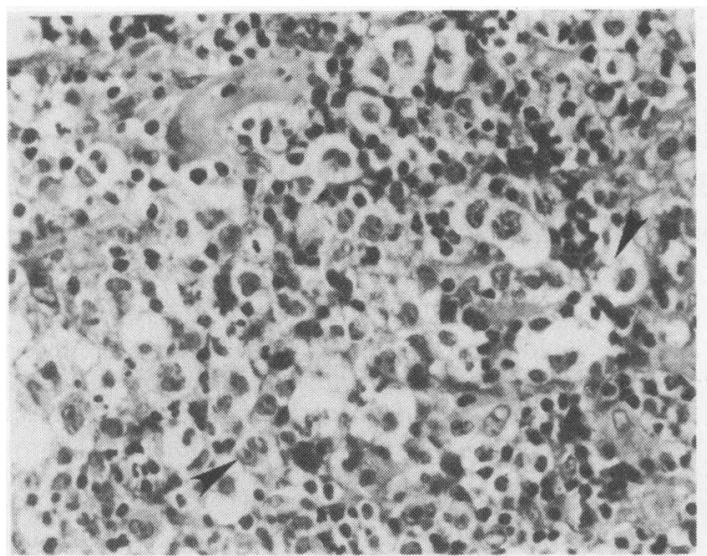

(a)

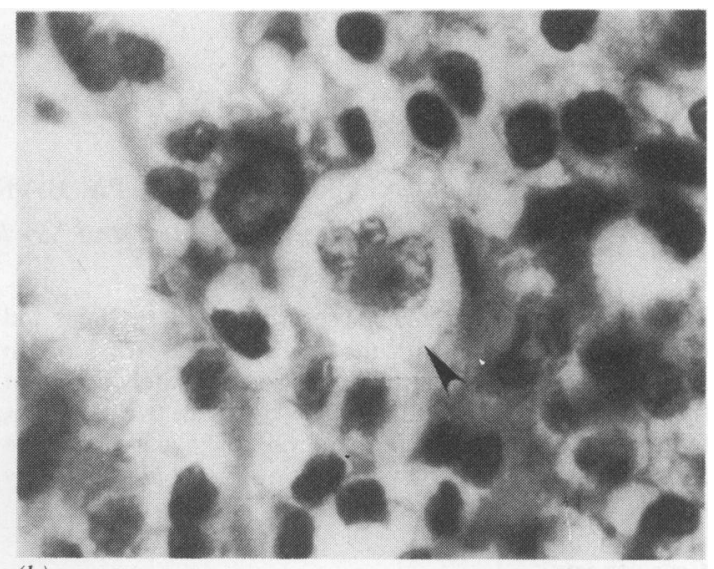

(b)

Fig 2 (a) Photomicrograph $(\times 280)$ showing several mononuclear variants of Reed-Sternberg cells (arrows) . (b) photomicrograph $(\times 640)$ showing Reed-Sternberg cell.

vincristine, procarbazine, and prednisone. $\mathrm{He}$ responded to treatment with resolution of his lung mass and is in remission.

\section{Discussion}

Intrathoracic Hodgkin's disease typically presents with bilateral but asymmetric mediastinal and hilar lymphadenopathy. Mediastinal adenopathy most often occurs in the paratracheal and bifurcation lymph nodes, followed closely by the bronchopulmonary nodes. The anterior mediastinal nodes are commonly affected but the posterior mediastinal nodes only rarely. Parenchymal disease most commonly occurs from direct extension from diseased nodes via lymphoid tissue of the bronchovascular sheath or through direct interstitial spread.

According to the theory of Wolpaw et al, the distribution of pulmonary disease reflects the distribution of lymphoid tissue within the lung. ${ }^{7}$ Lymphoid tissue is located primarily at points of bifurcation of bronchi and pulmonary vessels $^{68}$ and infiltration occurs largely along peribronchial and perivascular lymphatics. Peribronchial or endobronchial infiltrates (or both) have been reported to be the most common type of radiographically detected Hodgkin's disease affecting the lung, with direct invasion from hilar nodes and nodular or pneumonic infiltrates occurring less frequently. ${ }^{2}$

Kern and associates ${ }^{5}$ proposed criteria for establishing a diagnosis of primary pulmonary Hodgkin's disease, including: (1) anatomical documentation of typical pathological appearances; (2) restriction to the lung or with minimal disease of hilar nodes; and (3) adequate exclusion of disease at other lymph node sites. To our knowledge, this report documents the second case of primary pulmonary Hodgkin's disease described in English publications. ${ }^{4}$
This case report illustrates that although rare primary Hodgkin's disease of the lung is a distinct clinical entity. Nevertheless, when pulmonary infiltrates constitute the initial clinical presentation of Hodgkin's disease, an exhaustive effort to identify disease in other lymph node groups should be undertaken since this is most likely to be present and will alter staging and treatment.

Intrathoracic Hodgkin's disease can often be an elusive diagnosis. In Hodgkin's disease, in contrast to bronchogenic carcinoma, the peribronchial lymph nodes and bronchial walls are affected initially and bronchoscopically visible mucosal lesions occur late in the disease. As the major differential diagnosis is frequently bronchogenic carcinoma, which carries a drastically different clinical course and prognosis, vigorous diagnostic efforts are clearly warranted.

\section{References}

Ellman P, Browdler AJ. Pulmonary manifestations of Hodgkin's disease. Br J Dis Chest 1970);54:59-71.

McDonald JB. Lung involvement in Hodgkin's disease. Thorax 1977;32:664-7.

${ }^{3}$ Whitcomb ME, Schwarz MI, Keller AP, Flannery EP, Blom J. Hodgkin's disease of the lung. Am Rev Respir Dis 1972;106:79-85.

${ }^{4}$ Dhingra HK, Flance JI. Cavitary primary pulmonary Hodgkin's disease presenting as pruritis. Chest 1970);58:71-3.

${ }^{5}$ Kern WH, Crepeau AG, Jones JE. Primary Hodgkin's disease of the lung: report of four cases and review of the literature. Cancer 1961:14:1151-65.

"Anonymous. Case records of the Massachusetts General Hospital. N Engl J Med 1981;305:939-87.

7 Wolpaw SE, Higley CS, Havser H. Intrathoracic Hodgkin's disease. Am J Roentg 1944;52:374-87.

"Stolbert HO, Patt NL, MacEwen KF, Warwick OH, Brown IC. Hodgkin's disease of the lung: roentgenologic pathologic correlation. Am J Roentg 1964;92:96-115. 